\title{
Comparison of injection of midazolam-fentanyl with pethidine in management of pain induced by extracorporeal shock wave lithitripsy: A randomized clinical trial
}

\author{
Ahmad Kameli ${ }^{1}$, Ramin Haghighi ${ }^{2}$, Mahmoud Marhamati ${ }^{3}$, Mohammad Zarei ${ }^{4},{ }^{*}$
}

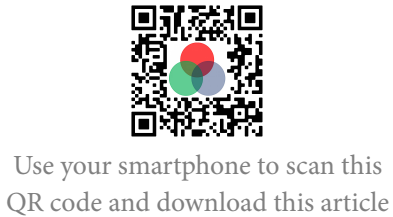

${ }^{1}$ Nursing Department, Faculty of Nursing, North Khorasan University of Medical Sciences, Bojnurd, Iran

${ }^{2} M D$, Department of Urology, Imam Hasan Hospital, North Khorasan University of Medical Sciences, Bojnurd, Iran

${ }^{3}$ Instructor of Medical Surgical Nursing, Department of Nursing, Esfarayen Faculty of Medical Sciences, Esfarayen, Iran

${ }^{4}$ Nursing Department, Shirvan Center of Higher Health Education, North Khorasan University of Medical Sciences, Bojnurd, Iran

Correspondence

Mohammad Zarei, Nursing Department, Shirvan Center of Higher Health Education, North Khorasan University of Medical Sciences, Bojnurd, Iran

Email: zareim51@gmail.com

History

- Received: Dec 21, 2019

- Accepted: Mar 05, 2020

- Published: Apr 29, 2020

DOI : 10.15419/bmrat.v7i4.599

\section{Check for updates}

\section{Copyright}

(-) Biomedpress. This is an openaccess article distributed under the terms of the Creative Commons Attribution 4.0 International license.

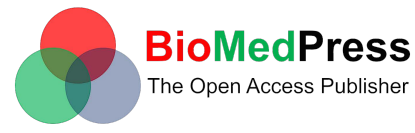

\begin{abstract}
Introduction: Urinary stones are the third most common disease of the genitourinary tract after urinary tract infections and prostate diseases. One of the ways to remove a urinary stone is extracorporeal shock wave lithitripsy, which crushes the stones for easier removal, and it is often used to reduce pain, reduce anxiety, and stabilize the patient. In this regard, the use of effective analgesics with less serious side effects seems reasonable. Methods: This randomized clinical trial study was performed with 90 patients who were divided into two groups according to a random number table. The first group received pethidine, and the second group received midazolam and fentanyl (midazolam-fentanyl). The type of medication used and demographic information were recorded, and the patients' pain was assessed by a visual analog pain scale at 15, 30, 45, and 60 minutes. Results: Of the subjects, 59 (65.5\%) were male and 30 (33.5\%) were female. The mean age of the patients was $38.4 \pm 13.5$ years. The level of pain at 15 minutes was $3.71 \pm 2.4$ in the midazolam-fentanyl group and $5.33 \pm 2.9$ in the pethidine group. At 45 minutes, the pain level was $4.2 \pm 3.1$ in the midazolam-fentanyl group and $5.26 \pm 2.72$ in the pethidine group. The differences between groups was significant at 15 and 45 minutes. At 30 and 60 minutes, the pain was lower in the midazolam-fentanyl group than in the pethidine group, but these differences were not statistically significant. There was no significant difference between the two groups with respect to the incidence of nausea and vomiting, restlessness, and anxiety. Conclusion: This study showed that the pain reported by patients using fentanyl-midazolam was lower than the pain reported by patients on pethidine, and the pain decreased with time in both groups. Therefore, if there is no other indication for the use of drugs, the combination of fentanyl-midazolam will have a better effect on pain and should be used. Trial registration: Current Controlled Trials IRCT2016051427893N1.

Key words: Pain, Extracorporeal shock wave lithotripsy, Pethidine, Midazolam
\end{abstract}

\section{INTRODUCTION}

Urinary stones are the third most common disease of the genital tract after urinary tract infections and prostate diseases ${ }^{1}$. The prevalence of these stones varies by age, sex, race, and geographic region. Its prevalence has been reported to be 1-15\% throughout a typical lifetime ${ }^{2}$. Stone removal is performed in several ways ${ }^{3}$; one of the most commonly used semi-invasive stone removal methods is extracorporeal shock wave lithotripsy to crush the stones. This is the treatment of choice in most urinary stones less than $20 \mathrm{~mm}$ wide ${ }^{4-6}$.

One of the most common complications of stone crushing is pain. Despite new technologies that have reduced pain, various medications have been used often to relieve pain, reduce anxiety, and reduce patient mobility during the procedure. Each of these medications has different advantages and disadvantages. Patients should have a short period of awak- ening after the procedure, and the use of anesthetics and long-acting analgesics should be limited. Therefore, the use of effective analgesics with fewer side effects and less drowsiness seems reasonable ${ }^{7}$. Some studies have not directly recommended the use of potent drugs, such as remifentanil, because of its respiratory effects ${ }^{8}$. Other studies have suggested using anesthetics, such as ketamine, that have lower respiratory effects at low doses ${ }^{9}$. Some studies have evaluated drugs like opioids, anesthetics, and non-steroidal anti-inflammatory drugs ${ }^{10,11}$. Despite this technique being widely used, it has no specific standard protocol for pain control, and opinions on the methods of pain medication and techniques for this operation vary widely ${ }^{3}$. Pain can cause the patient to move during stone crushing and disrupt the procedure. Reducing pain is important because pain can disrupt one's energy, affect one's ability to communicate and interact socially, and skew one's understanding of the 
meaning of life. The need to identify an effective way and suggest a more effective medication for pain management has motivated us to take steps to address this lack of a protocol. We conducted a study to compare the effects of pethidine and midazolam with general anesthesia on pain relief during extramedullary crushing.

\section{MATERIALS - METHODS}

This randomized clinical trial study was performed with 90 patients referred to the North Khorasan University of Medical Sciences (Iran) for lithotripsy. All patients were monitored for long-term opiate use, continuous analgesic use, and history of psychiatric disorders. Inclusion criteria included consent to participate in the research, reading and writing skills, being alert at the right level of communication, no longterm history of taking opioids and painkillers continuously, no history of mental disorders, high risk of anesthesia according to ASA charts (score of 1 or 2 ), and a history of allergic reactions to drugs (especially anesthesia drugs). Exclusion criteria included lack of consent to cooperate at any time, patients who were unable to work with the researcher (due to unconsciousness, severe hearing and speech problems, and inability to communicate), patients who for whatever reason required deeper anesthesia and other medications following lithotripsy, and patients who were contraindicated for opiate drugs or anesthetics (Figure 1 ).

If the patient needed a non-prescription medication during the operation, it was administered but then the patient was excluded from the study, and another patient was selected to replace him or her. The patients were divided into two groups of 45 with a random numbers table. The first group received $0.5 \mathrm{mg} / \mathrm{kg}$ pethidine (produced by Hameln) after the first injection, and the second group received midazolamfentanyl [midazolam (produced by Daroo Pakhsh) at 1 to $2 \mathrm{mg}$ dose and fentanyl (produced by Daroo Pakhsh) at 1 to $2 \mu \mathrm{g} / \mathrm{kg}$ dose]. An anesthesiologist performed the injections. All patients underwent expert anesthesia and standard monitoring throughout the duration of the lithotripsy. The lithotripsy was performed for all participants, and the voltage, time of lithotripsy, and frequency of exposure to the ultrasonic waves were recorded. The type of medication used and demographic information were also recorded in the relevant checklists, as well as patients' pain levels as assessed by a visual analog pain scale (VAS) at 15, 30, 45, and 60 minutes.

\section{ETHICAL CONSIDERATIONS}

This study was approved by the Medical Research and Ethical Committee of Esfarayen University of Medical Sciences (IR.ESFARAYENUMS.REC.1394.11).

\section{DATA ANALYSIS}

The completed patient checklists were coded and entered into SPSS 20 without anonymity. The study was blind in that pain assessors and statistical analyzers did not know the type of drug used or the group to which the patient belonged. An independent t-test was used to compare the pain in the two groups midazolam-fentanyl and pethidine - and to compare the differences between the nominal factors and the chi-square test.

\section{RESULTS}

In this study, 90 patients were divided into two groups ( $\mathrm{n}=45$ per group). Of the subjects, 59 (65.5\%) were male and 30 (33.5\%) were female. The mean age of the patients was $38.4 \pm 13.5$ years. The mean stone size was $11.14 \pm 3.5 \mathrm{~mm}$. Of the subjects, $74(82.2 \%)$ had no history of the disease, 5 (5.6\%) had hypertension, and $6(6.7 \%)$ had diabetes (Table 1$)$.

The pain level measured by the VAS in the midazolam-fentanyl group was $3.71 \pm 2.4$ and in the pethidine group was $5.33 \pm 2.9$ at 15 minutes post-operation. The pain level reported by the midazolam-fentanyl group was significantly lower $(\mathrm{p}=0.006)$. At 30 minutes, the mean pain was 4.33 \pm 3.10 in the midazolam-fentanyl group and $5.11 \pm$ 2.72 in the pethidine group. This difference in mean pain was not significant, despite the decrease found in the midazolam-fentanyl group. The level of pain on the VAS at 45 minutes after lithotripsy was 4.2 \pm 3.1 in the midazolam-fentanyl group and $5.26 \pm$ 2.72 in the pethidine group (pain was significantly higher in the pethidine group). At 60 minutes, the mean pain score in the midazolam-fentanyl group was significantly lower than that of the pethidine group, but this difference was not statistically significant (Table 2). Analysis of variance with repeated measurements was used, and it showed that the rate of pain decreased significantly over time $(p=0.001)$ (Figure 2). The incidences of nausea, restlessness, and vertigo (i.e., differences in complications) for the two groups were not significant according to the Chi-square test (Table 3).

\section{DISCUSSION}

Extracorporeal shock wave lithotripsy is one of the most common operations for breaking and removing urinary stones. Pain relief seems necessary, due 


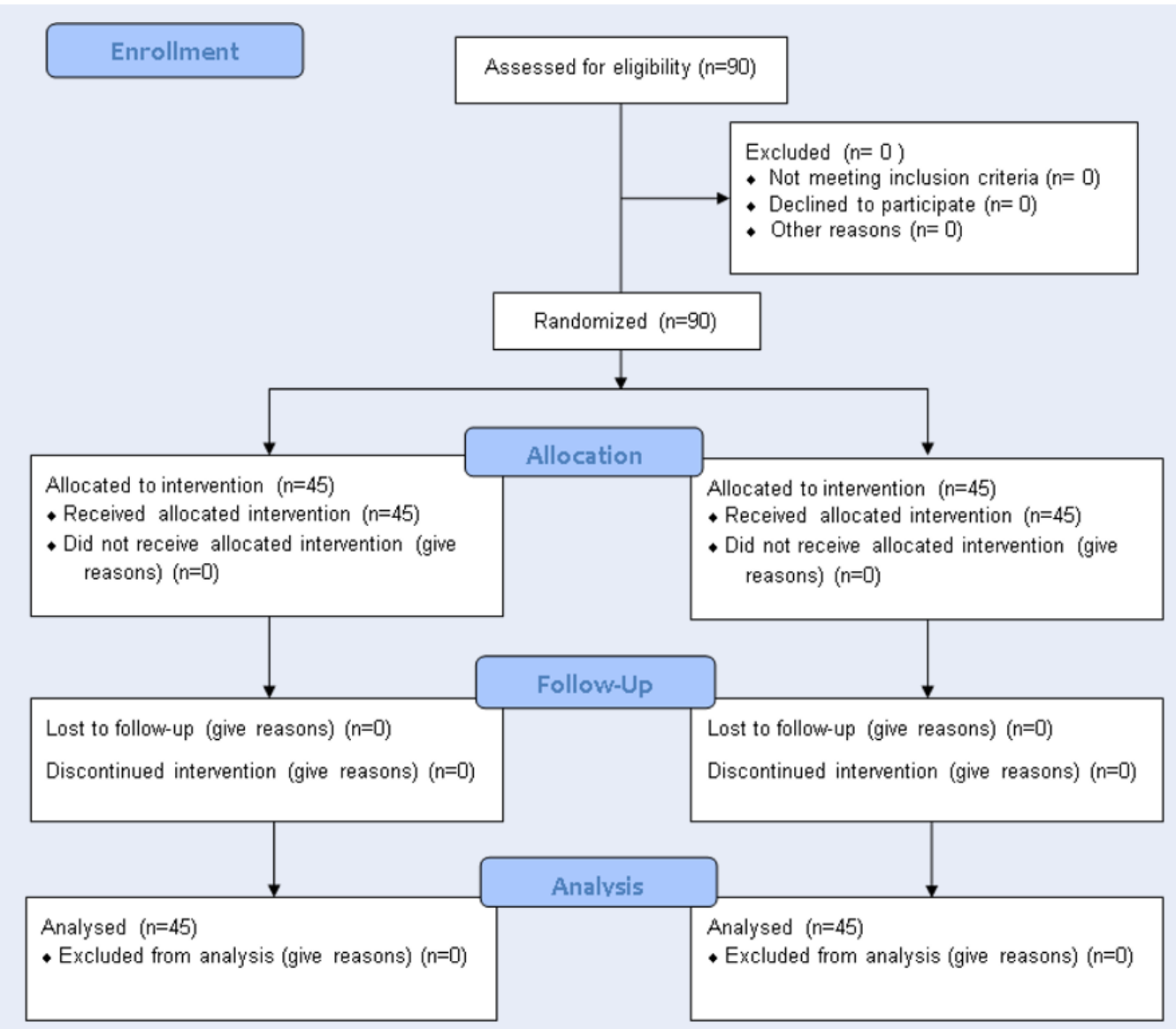

Figure 1: Consort flow diagram of the study.

Table 1: Demographic characteristics and comparisons between the two groups

\begin{tabular}{lccc}
\hline Variable & Group 1 (Fentanyl-midazolam) & Group 2 (Pethidine) & P-value \\
\hline Age & $43.07 \pm 41.14$ & $32.42 \pm 35.12$ & 0.042 \\
Size of stone in millimeters & $30.20 \pm 11.3$ & $98.90 \pm 10.30$ & 0.67 \\
$\begin{array}{l}\text { The number of shock waves } \\
\text { given }\end{array}$ & $36.80 \pm 3561.66$ & $44.17 \pm 3544.44$ & 0.89 \\
Voltage used & $27.90 \pm 72.30$ & & \\
\hline
\end{tabular}

Table 2: VAS pain scores and comparison between groups at different times

\begin{tabular}{lccc}
\hline Variable & $\begin{array}{c}\text { Group 1 } \\
\text { (Fentanyl-midazolam) }\end{array}$ & Group 2 (Pethidine) & P-value \\
\hline Pain at 15 minutes & $2.42 \pm 3.71$ & $2.96 \pm 5.33$ & $\mathbf{0 . 0 0 6}$ \\
Pain at 30 minutes & $3.10 \pm 4.33$ & $2.72 \pm 5.26$ & $\mathbf{0 . 1 3}$ \\
Pain at 45 minutes & $2.96 \pm 4.2$ & $2.59 \pm 5.37$ & $\mathbf{0 . 0 4 8}$ \\
Pain at 60 minutes & $3.11 \pm 3.53$ & $2.24 \pm 4.15$ & $\mathbf{0 . 3 6}$ \\
\hline
\end{tabular}




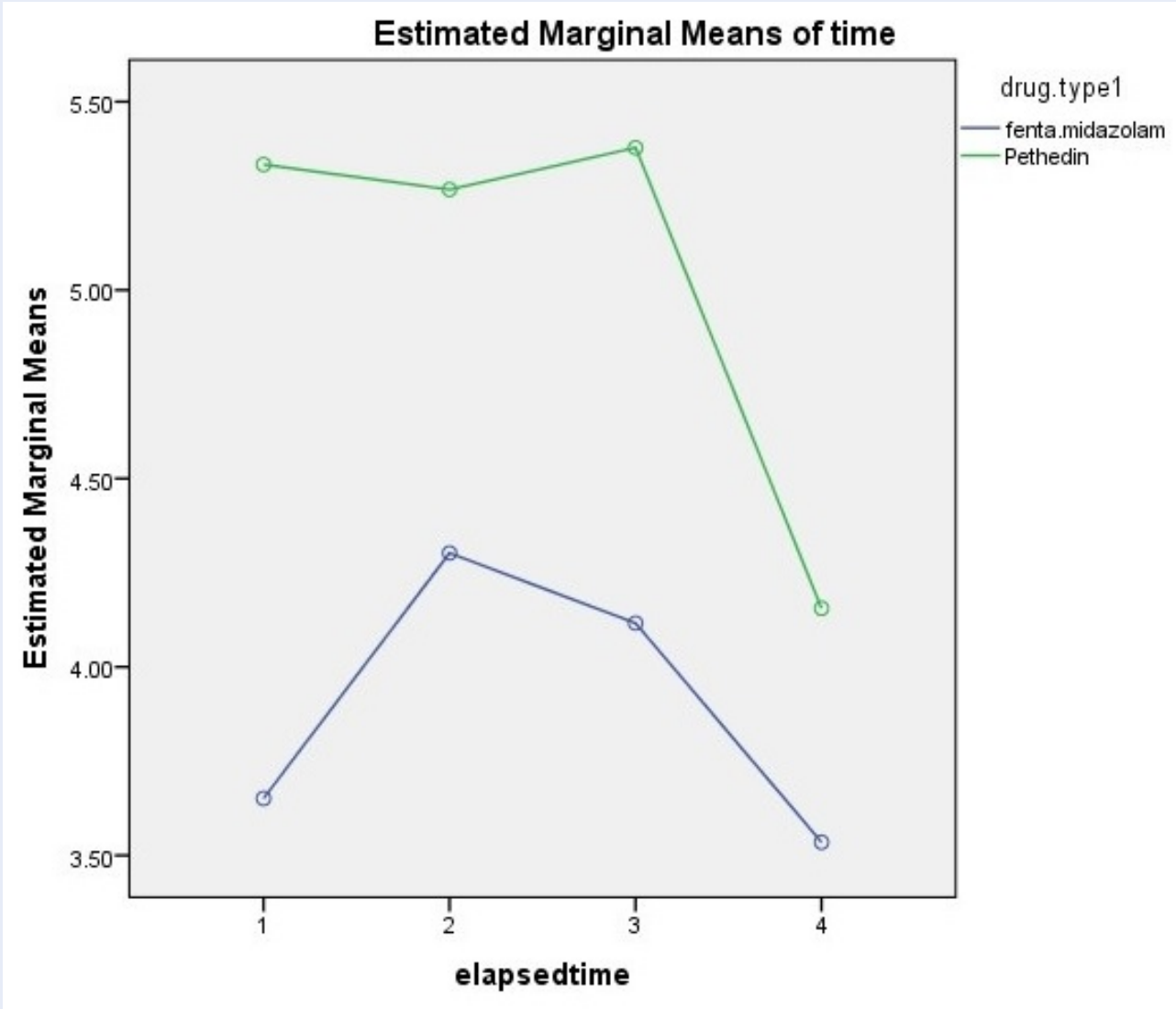

Figure 2: The amount of pain changes over time.

Table 3: Comparison of the incidence of postoperative complications in the two groups

\begin{tabular}{lccccc}
\hline Type of complication & \multicolumn{2}{c}{ Pethidine } & Fentanyl-midazolam & P-value \\
\hline & No & Yes & No & Yes & \\
Nausea & 40 & 5 & 9 & 5 & 0.97 \\
Restlessness & 43 & 0 & 43 & 1 & 0.32 \\
Vertigo & 30 & 15 & 21 & 24 & 0.056 \\
\hline
\end{tabular}

to the patient's discomfort during stone crushing and inability to move, but anesthesia is not common for this purpose. Therefore, injectable, topical, and other painkillers are recommended. Medication with opiates is usually preferred because they guarantee patient comfort with easy recovery and fewer side effects $^{12}$. Therefore, finding a suitable way to reduce pain can help patients and therapists. Pethidine and midazolam-fentanyl were used to reduce pain in both groups. The mean pain reduction at 15 and 45 minutes in the midazolam-fentanyl group was significantly different from that in the pethidine group. A VAS was used for pain measurement, and the re- sults showed that the pain intensity in the midazolamfentanyl group was less than that in the pethidine group at 20 minutes and 2 hours, but this difference was not statistically significant ${ }^{4}$.

In our study, there was a decrease in pain severity over time, but the difference between the two groups was significant only at 45 and 15 minutes. However, it is noted that although Mehrabi et al. used opiate analgesics, their drug combination was a little different from ours. In a 2013 study conducted by Lee et al. at the Center for Urology in China, three drugs were assessed for pain relief from crushing shock: a sodium diclofenac analgesic, a eutectic mixture of lo- 
cal anesthetic, and a diclofenac gel. The organs were compared in the study and there were no significant differences found among the three drugs ${ }^{13}$. Batch et al. published a review article entitled "Drugs for pain management in shock wave lithotripsy". In that study, drug use was introduced as one of the best ways to control pain in patients using shock wave lithotripsy ${ }^{3}$. This is in line with the reduction of pain in the two groups in our study. A study by Alibeigi et al. compared the effects of piroxicam and pethidine $^{2}$. In a study by Ezkan et al., midazolam, fentanyl, diclofenac, and tramadol were used for pain management after extracorporeal shock wave lithotripsy; their study found a preference for diclofenac and tramadol over midazolam and fentanyl ${ }^{14}$. Our study used two types of opioids. In their study of nausea, Demir et al. made a comparison of pethidine and diazepam with diclofenac and hyoscine, and found that pethidine and diazepam had a greater effect on pain relief than did diclofenac and hyoscine. With respect to vomiting, their study did not observe a difference between the two groups. Likewise, in our study, nausea and vomiting (and other complications like dizziness and restlessness) were not significantly different between the two groups. Moreover, Demir et al. used pethidine and compared it to a non-opioid drug; there were no significant differences also in their study with respect to side effects like nausea and vomiting ${ }^{15}$.

The main limitations of our study were the confined location of the study and the prolonged sampling procedure.

\section{CONCLUSION}

This study showed that the lithotripsy pain reported by patients using fentanyl-midazolam was lower than that reported by patients using pethidine, and that pain decreased with time in both patient groups. Therefore, if there is no other indication for the use of drugs, the combination of fentanyl and midazolam will probably have a better effect on pain, and should be considered for pain management.

\section{ABBREVIATIONS}

ESWL: Extracorporeal shock wave lithotripsy

\section{ACKNOWLEDGMENTS}

This article was derived from a research project approved by the Esfarayen Faculty of Medical Sciences, which was conducted with the financial support of this university. The researchers would like to express their gratitude to the Faculty Research Council and all patients, who participated in this study.

\section{AUTHOR'S CONTRIBUTIONS}

M.Z., A.K. and H.M. contributed to the conceptualization and design of the study, the acquisition, analysis and interpretation of data. M.Z. and R.Z. were for drafting the article and revising the article critically for important intellectual content. All authors read and approved the final manuscript.

\section{FUNDING}

This article was derived from a research project approved by the Esfarayen Faculty of Medical Sciences, which was conducted with the financial support of this faculty.

\section{AVAILABILITY OF DATA AND MATERIALS}

Data and materials used and/or analysed during the current study are available from the corresponding author on reasionable request.

\section{ETHICS APPROVAL AND CONSENT TO PARTICIPATE}

This study was conducted in accordance with the amended Declaration of Helsinki. The institutional review board (Medical Research and Ethical Committee of Esfarayen Faculty of Medical Sciences) approved the study (IR.ESFARAYENUMS.REC.1394.11), and all participants provided written informed consent.

\section{CONSENT FOR PUBLICATION}

The authors hereby consents that the Publisher publishes the Work.

\section{COMPETING INTERESTS}

The authors declare that they have no competing interests.

\section{REFERENCES}

1. Masood E, Majid M, MoamadAli Z, Artin K. The results of transuretral lithotripsy of ureteral stones in Hashemi Nejad Hospital. Journal Iran University of Medical Sciences [In Persian]. 2001;8(10):817-821.

2. Alibeigi FM, MoazeniBistgani M. Comparison efficacy of topical piroxicam gel and lidocaine with intravenous pethidine in reducing pain during ESWL. Armaghane danesh [In Persian]. 2011;16(2).

3. Bach C, Zaman F, Kachrilas S, Kumar P, Buchholz N, Masood J. Drugs for pain management in shock wave lithotripsy. Pain res treat. 2011;259426. PMID: 22135735. Available from: https: //doi.org/10.1155/2011/259426.

4. Mehrabi S, Shirazi KK, Hadinia A. Comparison of fentanyl and combination of pethedine and midazolam for pain control during extracorporeal shock wave lithotripsy. Shahrekord University of Medical Sciences [Original article] [In Persian] 2011;13(3):70 -76. 
5. Putman SS, Hamilton BD. The use of shock wave lithotripsy for renal calculi. Curr opin urol. 2004;14(2):117-121. PMID 15075841. Available from: https://doi.org/10.1097/00042307200403000-00012.

6. Al-Marhoon MS, Shareef O, Al-Habsi IS, Balushi ASA, Mathew J, Venkiteswaran KP. Extracorporeal shock-wave lithotripsy success rate and complications: Initial experience at Sul$\tan$ Qaboos University Hospital. Oman medical journal. 2013;28(4):255-259. Available from: https://doi.org/10.5001/ omj.2013.72PMid:23904918.

7. Medina HJ, Galvin EM, Dirckx M, Banwarie P, Ubben JF, Zijlstra $\mathrm{FJ}$, et al. Remifentanil as a single drug for extracorporeal shock wave lithotripsy: A comparison of infusion doses in terms of analgesic potency and side effects. Anesthesia and analgesia. 2005;101(2):365-370. PMID: 16037145. Available from: https: //doi.org/10.1213/01.ANE.0000159379.54705.84.

8. Mallick A, Elliot S. Remifentanil is too potent to be given by bolus. British journal of anaesthesia. 2004;93(2):305-306. PMID: 15251998. Available from: https://doi.org/10.1093/bja/aeh589.

9. Gharaei B, Jafari A, Aghamohammadi H, Kamranmanesh M, Poorzamani $M$, Elyassi $H$, et al. Opioid-sparing effect of preemptive bolus low-dose ketamine for moderate sedation in opioid abusers undergoing extracorporeal shock wave lithotripsy: A randomized clinical trial. Anesthesia and analgesia [In Persian] . 2013;116(1):75-80. Available from: https: //doi.org/10.1213/ANE.0b013e31826f0622PMid:23223117.

10. Eker HE, Cok OY, Ergenoglu P, Aribogan A, Arslan G. IV paracetamol effect on propofol-ketamine consumption in paediatric patients undergoing ESWL. Journal of anesthesia.
2012;26(3):351-356. PMID: 22349746. Available from: https: //doi.org/10.1007/s00540-012-1335-4.

11. Ozcan S, Yilmaz E, Buyukkocak U, Basar H, Apan A. Comparison of three analgesics for extracorporeal shock wave lithotripsy. Scandinavian journal of urology and nephrology. 2002;36(4):281-285. PMID: 12201921. Available from: https: //doi.org/10.1080/003655902320248254.

12. Daly KM, Chaker K, Rhouma SB, Chehida MAB, Ouanes Y, Sellami $A$, et al. Evaluation of protocols on the use of analgesics for pain management in patients with extracorporeal shock wave lithotripsy (ESWL). Pan African medical journal. 2019;32:109. PMID: 31223399. Available from: https://doi.org/ 10.11604/pamj.2019.32.109.18211.

13. Liu J, Zang YJ. Comparative study between three analgesic agents for the pain management during extracorporeal shock wave lithotripsy. Urol J. 2013;10(3):942-945.

14. Ozcan S, Yilmaz E, Buyukkocak U, Basar H, Apan A. Comparison of three analgesics for extracorporeal shock wave lithotripsy. Scandinavian journal of urology and nephrology. 2002;36(4):281-285. PMID: 12201921. Available from: https: //doi.org/10.1080/003655902320248254.

15. Demir A, k Cecen K, Karadag MA, Uslu M, Arslan OE. Pain control using pethidine in combination with diazepam compared to diclofenac in combination with hyoscine-n-butyl bromide: in patients undergoing extracorporeal shock wave lithotripsy. Central European journal of urology. 2015;68(2):201-206. PMID: 26251744. Available from: https://doi.org/10.5173/ceju. 2015.479 . 